\title{
World Patient Safety Day 2021: "Safe Maternal and New Born Care”
}

\author{
Geetha Balsarkar ${ }^{1}$
}

Received: 9 August 2021 / Accepted: 9 August 2021 / Published online: 23 August 2021

(c) Federation of Obstetric \& Gynecological Societies of India 2021

Patient harm unknowingly or knowingly due to adverse uncertain events occurring while in hospital is one of the leading causes of morbidity and mortality worldwide [1]. It is very significant in obstetric practice. Conditions like wrong drug administered to wrong patient, anaphylactic reactions to certain drugs, post-partum haemorrhage due to certain conditions and delayed decision-making are all contributing to patient harm.

Patient safety is a new health care discipline that has emerged due to the growing complexity of health care systems with multiple employees involved and the rise of patient harm in health care facilities [2]. In hospitalization in low- and middle-income countries, there are 134 million adverse events annually, which in turn lead to 2.6 million deaths. In high-income countries, approximately one in ten patients is harmed while receiving hospital care [3]. These can be easily avoided with a little diligence.

This global campaign of World Patient Safety Day is the result of a series of annual Global Ministerial Summits on Patient Safety in London and Bonn initiated from 2016, as well as the high-level advocacy and commitment of major international and national stakeholders in patient care and management. World Patient Safety Day was established in 2019 by the Seventy-second World Health Assembly through the adoption of resolution WHA 72.6-"Global action on patient safety" [4]. The Day is firmly grounded in the fundamental principle of medicine, Primum non nocere-first do no harm. The overall objectives of the Day celebrations

Dr. Geetha Balsarkar is a Professor at Seth G.S. Medical College and Nowrosjee Wadia Maternity Hospital, Mumbai, India. She is a Librarian of Mumbai Obstetrics Gynecology Society, President of Menopause Society, Mumbai Branch, President of AMWI, Mumbai Branch, and Editor-In-Chief, Journal of Obstetrics and Gynecology of India, FOGSI Journal.

Geetha Balsarkar

gdbalsarkar@yahoo.com

1 Department of Obstetrics and Gynecology, Seth G. S. Medical College, Nowrosjee Wadia Maternity Hospital, Mumbai, India are to enhance global awareness of patient safety, increase public engagement in health care safety, and promote global action to prevent and reduce avoidable harm in health care. Each year a different theme is selected to shed light on a priority area critical to patient safety and ultimately the achievement of universal health coverage.

In 2019, the theme for the very first World Patient Safety Day was "Patient Safety: A Global Health Priority". To promote open communication for learning from errors and to emphasize the importance of everyone's voice in prioritizing patient safety, the slogan was "Speak up for patient safety!". It encouraged even junior doctors and nurses to communicate in clear language if they found any decision or action detrimental to the patient. World Patient Safety Day 2020 focuses on the interrelationship between health worker safety and patient safety. It could not have been more appropriate with the advent of COVID-19. The slogan, "Safe health workers, Safe patients", emphasizes the need for a safe working environment for health workers as a prerequisite for ensuring patient safety. Along with this slogan, WHO is proposing the following call for action: "Speak up for health worker safety!" [5, 6]. Health Worker Safety Charter and World Patient Safety Day Goals 2020 were launched on 17 September 2020 to call for action to improve health worker safety globally [7].

The theme selected for World Patient Safety Day 2021 is "Safe maternal and new born care", due to the significant burden of risks and harm women and newborns are exposed to when receiving care during childbirth. The issue has been further compounded multiple times by the COVID-19 pandemic, which has led to the disruption of essential health services due to breaks in supply chains, women's inability to access care, and the shortage of skilled health professionals. Even before the pandemic, approximately 810 women and 6700 newborns died daily, with the causes occurring mainly around the time of childbirth. Despite the significant progress made in reducing maternal and neonatal mortality, the SDG 3 targets are still far from being achieved [8].

About 2 million babies were also stillborn every year, with over $40 \%$ occurring during labour. Most of these deaths 
and stillbirths are avoidable through the provision of safe and quality care by skilled health care professionals working in supportive environments. Since maternity care is also affected by issues of gender equity and violence, women's experiences during childbirth have the potential to either empower or to inflict damage and emotional trauma on them. Therefore, this year's campaign slogan-"Act now for safe and respectful childbirth!"-calls on all stakeholders to accelerate the actions necessary for ensuring safe and respectful childbirth. It is envisaged that the momentum generated by World Patient Safety Day 2021 will revamp stakeholders' efforts to achieve universal health coverage for all women and newborns and ultimately achieve the maternal and newborn SDG 3 targets [8].

Objectives of the World Patient Safety Day 2021 are:

1. Raise global awareness on the issues of maternal and newborn safety, particularly during childbirth.

2. Engage multiple stakeholders and adopt effective and innovative strategies to improve maternal and newborn safety.

3. Call for urgent and sustainable actions by all stakeholders to scale up efforts, reach the unreached and ensure safe maternal and new born care, particularly during childbirth.

4. Advocate the adoption of best practices at the point of care to prevent avoidable risks and harm to all women and newborns during childbirth [1].

The obstetric care is compounded by the disruption of essential health services caused by the COVID-19 pandemic; hence, the campaign is even more important this year.

Fortunately, the majority of stillbirths and maternal and newborn deaths are avoidable through the provision of safe and quality care by skilled health professionals working in supportive environments. This can only be achieved through the engagement of all stakeholders and the adoption of comprehensive health systems and community-based approaches.

COVID-19 makes achieving optimal patient flow more important than ever. Optimal patient flow for safety has never been more critical than in the COVID era. Dramatic expansion of emergency departments, acute care, and critical care units to meet the anticipated needs of COVID-19 positive patients has been essential to provide safe and effective care for patients in crisis.

During the continuing challenges of the COVID-19 pandemic, a combination of virtual and other activities are being planned by WHO to observe and celebrate the Day in September 2021. The signature mark of the global campaign is to light up iconic monuments, landmarks, and public places in the colour orange [1].

WHO calls upon all stakeholders-governments, nongovernmental organizations, professional organizations, civil society, patient organizations, academia and research institutes - to join the global campaign by lighting up iconic monuments in orange, and organizing international, national and local activities and events on and around 17 September 2021.

"ACT NOW FOR SAFE AND RESPECTFUL CHILDBIRTH!"

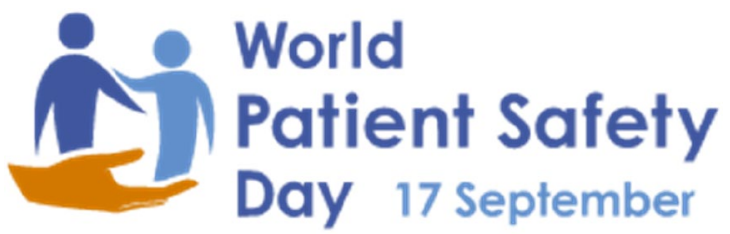

It is all about getting global health care actors involved in:

- Scaling up concerted global action on patient safety.

- Putting measures in place to reduce avoidable patient harm.

- Sharing awareness in patient safety in care settings.

- Raising awareness amongst the public and media on key issues relating to patient safety.

- Experimenting new ideas to improve patient safety.

- Helping patients to contribute to their own safety [1].

\section{References}

1. "World Patient Safety Day".

2. "Patient Safety" WHO fact sheet. September 2019.

3. "The economics of patient safety" OECD.

4. "WHA Resolution 72.6 'Global Action on Patient Safety'. World Health Organization." World Health Organization (WHO).

5. "World Patient Safety Day 17 September 2020".

6. "Protect health workers to save patients, WHO reiterates on World Patient Safety Day".

7. "World Patient Safety Day 17 September 2019".

8. "Campaigns" World Health Organization (WHO).

Publisher's Note Springer Nature remains neutral with regard to jurisdictional claims in published maps and institutional affiliations. 


\section{About the Author}

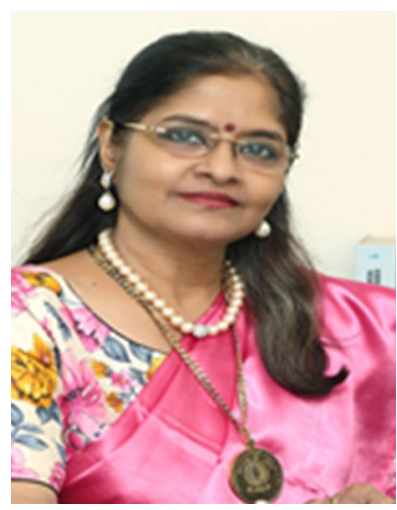

Dr. Geetha Balsarkar is a Professor at Seth G.S. Medical College and Nowrosjee Wadia Maternity Hospital, Mumbai, India. She is a Librarian of Mumbai Obstetrics Gynecology Society, President of Menopause Society, Mumbai Branch, President of AMWI, Mumbai Branch, and Editor-InChief, Journal of Obstetrics and Gynecology of India, FOGSI Journal. She has undergone training for the PPIUCD program and is also the faculty member of many training programmes as well. She has over 18 years of teaching experience and is actively working on FIGO FOGSI Project of PPIUCD in India. She is a recipient of the prestigious Hargobind Foundation for the study of Fetal Medicine in 2008 at St. George's Hospital, London. 